\title{
Magnetic-field-induced orientational order in the isotropic phase of hard colloidal platelets
}

\author{
D. van der Beek, ${ }^{1, *}$ A. V. Petukhov, ${ }^{1}$ P. Davidson, ${ }^{2}$ J. Ferré, ${ }^{2}$ J. P. Jamet, ${ }^{2}$ H. H. Wensink, ${ }^{3}$ G. J. Vroege, ${ }^{1}$ W. Bras, ${ }^{4}$ and \\ H. N. W. Lekkerkerker ${ }^{1}$ \\ ${ }^{1}$ Van't Hoff Laboratory for Physical and Colloid Chemistry, Debye Institute, Utrecht University, \\ Padualaan 8, 3584 CH Utrecht, The Netherlands \\ ${ }^{2}$ Laboratoire de Physique des Solides, UMR 8502 CNRS, Université Paris-Sud, Bâtiment 510, 91405 Orsay, France \\ ${ }^{3}$ Institut für Theoretische Physik II, Heinrich-Heine-Universität Düsseldorf, \\ Universitätsstrasse 1, Gebäude 25.32, 40225 Düsseldorf, Germany \\ ${ }^{4}$ Netherlands Organisation for Scientific Research (NWO), DUBBLE CRG, European Synchrotron Radiation Facility, \\ BP 220, 38043 Grenoble Cedex, France \\ (Received 14 November 2005; published 5 April 2006)
}

\begin{abstract}
The magnetic-field-induced orientational order in the isotropic phase of colloidal gibbsite $\left[\mathrm{Al}(\mathrm{OH})_{3}\right]$ platelets is studied by means of optical birefringence and small-angle x-ray scattering (SAXS) techniques. The suspensions display field-induced ordering at moderate field strengths (a few Tesla), which increases with increasing particle concentration. The gibbsite particles align their normals perpendicular to the magnetic field and hence possess a negative anisotropy of their diamagnetic susceptibility $\Delta \chi$. The results can be described following a simple, Onsager-like approach. A simplified model is derived that allows one to obtain the orientational distribution function directly from the scattering data. However, it leads to an underestimate of the diamagnetic susceptibility anisotropy $\Delta \chi$. This accounts for the difference between the $\Delta \chi$ values provided by the two experimental techniques (SAXS and magneto-optics). The order of magnitude $\Delta \chi \sim 10^{-22} \mathrm{~J} / \mathrm{T}^{2}$ lies in between that of goethite suspensions and that of suspensions of organic particles.
\end{abstract}

DOI: 10.1103/PhysRevE.73.041402

PACS number(s): 82.70.Dd, 61.30.Gd, 61.10.Eq, 78.20.Fm

\section{INTRODUCTION}

Recently, gibbsite proved to be very suitable as a platelike model colloid. Its plates are quite monodisperse and easy to synthesize [1,2]. Suspensions of sterically and chargestabilized colloidal gibbsite platelets were found to display for the first time both nematic and columnar liquid crystals phases [3-7]. In this paper we present a study of the isotropic phase of colloidal gibbsite $\left[\mathrm{Al}(\mathrm{OH})_{3}\right]$ platelets dispersed in toluene submitted to a magnetic field. It is found that these particles display considerable orientational order at moderate magnetic fields. This in turn makes them highly suitable for liquid crystal studies in external magnetic fields.

The study of colloidal suspensions submitted to magnetic fields dates back to the beginning of the 20th century when John Kerr reported magnetic birefringence in a suspension of chemically precipitated magnetite [8]. Majorana [9] and Cotton and Mouton [10] studied magnetic birefringence in suspensions of so-called "fer Bravais," a suspension of iron oxides that was commonly used in pharmacies at that time. Cotton and Mouton furthermore found that pure liquids also showed magnetic birefringence [11], a phenomenon that is nowadays known by the discoverers' names.

In recent years, Lemaire and co-workers [12-14] have studied suspensions of colloidal goethite $(\alpha-\mathrm{FeOOH})$ nanorods that, as a matter of fact, are closely related to the sus-

\footnotetext{
* Present address: Laboratoire de Chimie de la Matière Condensée, Université Pierre et Marie Curie (Paris VI), 4 Place Jussieu, Case Courrier 174, 75252 Paris Cedex 05, France. Electronic address: vdbeek@ccr.jussieu.fr
}

pensions studied by Majorana and Cotton and Mouton. Suspensions of these rods, somewhat comparable to ferrofluids, are very sensitive to an external magnetic field. In their nematic liquid-crystalline phase, these suspensions align in fields as low as $0.03 \mathrm{~T}$. In addition, they display, in both isotropic and nematic phases, a very peculiar phenomenon, i.e., the reorientation of the rods with increasing field strength. Below about $0.35 \mathrm{~T}$, the rods align parallel to the field, whereas, above this value, they reorient perpendicularly.

As opposed to these suspensions of mineral rods, isotropic suspensions of organic particles, such as virus particles, need much higher fields (up to $20 \mathrm{~T}$ ) to display appreciable magneto-optical effects. Examples include rodlike cellulose microcrystals [15], tobacco mosaic virus (TMV) [16,17], fd virus $[18,19]$, and $P f 1$ virus [20] particles, as well as platelike biological membranes [21]. Although the shape of those particles is highly anisotropic, their intrinsic diamagnetic susceptibility anisotropy $\Delta \chi_{0}$ (i.e., of the bulk material) is much lower, giving rise to a much lower "effective" diamagnetic susceptibility anisotropy per particle $\Delta \chi$ than, e.g., inorganic goethite rods.

Here, we measure the linear birefringence as well as the small-angle $\mathrm{x}$-ray scattering (SAXS) to investigate the magnetic-field-induced order in the isotropic phase. Measurement of the linear birefringence is a well-known technique that gives information about the field-induced orientational order and that has been employed before with various kinds of liquid crystals. Examples of these include the classic thermotropic liquid crystals 5CB [22] and MBBA [23]. Among the lyotropic liquid crystals are (bio) polymers such as DNA [24] and hard rods such as the aforementioned TMV $[17,25], f d[18,19]$ virus particles, and goethite rods $[12,13]$. 


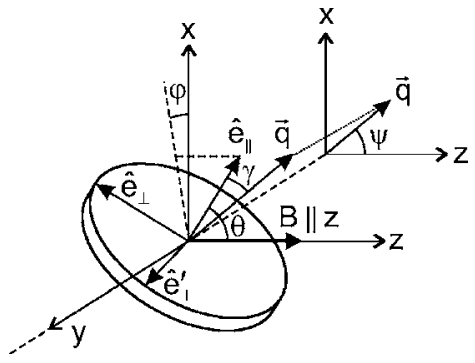

FIG. 1. Sketch of the magnetic and scattering coordinate system. The symbols are explained in the text.

Although measurement of the birefringence is a very sensitive technique, it only provides the second moment of the orientational distribution function, namely the nematic order parameter $S_{2}$. The SAXS measurements, however, can be analyzed with a simplified model that relates the orientational distribution function directly to the scattering data. In either way, we finally obtain the diamagnetic susceptibility anisotropy.

\section{THEORY}

\section{A. The orientational distribution function}

Let us consider a single platelike particle immersed in a magnetic field with magnitude $B$ directed along the $z$-axis. The orientation of the platelet is indicated by the platelet normal $\hat{e}_{\|}$, which makes an angle $\theta$ to the magnetic field direction (the $z$-axis), see Fig. 1 . For a mineral crystal-like gibbsite, the magnetic susceptibility $\chi$, and therefore the magnetization, will be different along the different axes of the particle. The magnetic energy can then be written as

$$
w_{\text {mag }}(\theta)=-\frac{1}{3} \Delta \chi B^{2} P_{2}(\cos \theta),
$$

where we have introduced the diamagnetic susceptibility anisotropy $\Delta \chi \equiv \chi_{\|}-\chi_{\perp}$ and the second order Legendre polynomial $P_{2}(x)=\frac{1}{2}\left(3 x^{2}-1\right)$, and where we have retained the orientation-dependent term only. In our case, as will be shown later on, $\Delta \chi$ is negative. Hence, in a magnetic field, the platelets tend to align with their normal $\hat{e}_{\|}$perpendicular to the field, as the magnetic energy is lowest in this configuration. The orientational distribution function (ODF) $f(\theta)$ of interacting platelets in a magnetic field can be found using an Onsager-like approach, i.e., by minimizing the free-energy expression containing the orientational entropy, packing entropy, and magnetic energy. For a weakly perturbed state, the ODF can be written as $f(\theta)=\frac{1}{4 \pi}\left[1+\varepsilon P_{2}(\cos \theta)\right]$ (with $|\varepsilon|$ $\ll 1$ ) and following Straley [26] it can be shown that

$$
f(\theta)=\frac{1}{4 \pi}\left[1+\frac{1}{3} \frac{\Delta \chi B^{2}}{k T} \frac{1}{1-\phi / \phi^{*}} P_{2}(\cos \theta)\right] .
$$

Here, $k$ is Boltzmann's constant, $T$ the temperature, $\phi$ the volume fraction, and $\phi^{*}$ the so-called spinodal volume fraction above which the isotropic state becomes locally unstable. The order parameter, defined as $S_{2} \equiv\left\langle P_{2}(\cos \theta)\right\rangle$, is given by

$$
S_{2}=\frac{1}{15} \frac{\Delta \chi B^{2}}{k T} \frac{1}{1-\phi / \phi^{*}},
$$

in agreement with earlier results $[17,26]$. This result is valid for both rod- and plate-like particles.

\section{B. Magnetic-field-induced birefringence}

A common way to measure the magnetic-field-induced orientational order, quantified by $S_{2}$, is by measuring the birefringence $\Delta n[13,16,18,22-25,27]$. It has been suggested that the birefringence of a dilute isotropic colloidal suspension is given by $[13,16,28]$

$$
\Delta n=\Delta n_{\text {sat }} \phi S_{2},
$$

where $\Delta n_{\text {sat }}$ is the specific birefringence, $\phi$ the volume fraction, and $S_{2}$ the order parameter. Note that, in this notation, the specific birefringence does not depend either on the concentration, or on the magnitude and direction of alignment of the particles. Combining Eqs. (3) and (4) yields

$$
\frac{\Delta n}{B^{2}}=\frac{1}{15} \frac{\Delta n_{\mathrm{sat}} \Delta \chi}{k T} \frac{\phi}{1-\phi / \phi^{*}},
$$

which relates the observed field-induced birefringence $\Delta n / B^{2}$ to the magnitude of $\Delta n_{\text {sat }} \Delta \chi$. Apparently, we may expect a linear dependence of $\Delta n / B^{2}$ vs $\phi$ at low volume fractions $\left(\phi / \phi^{*} \ll 1\right)$ when there are only individual interactions of the platelets with the magnetic field, while it should diverge on approaching the spinodal concentration $\phi^{*}$ due to the onset of collective interactions between the platelets.

\section{Small-angle $x$-ray scattering}

Extracting the nematic order parameter from the anisotropic SAXS patterns of aligned assemblies of anisotropic particles is a rather difficult matter that was already addressed in a number of studies dealing with various situations [29]. In this section, a very simple model is derived in order to analyze the SAXS results for this purpose. In general, the X-ray scattering intensity $I(\vec{q})$ of a suspension of particles is determined not only by the scattering of a single particle, i.e., the form factor $F$, but also by the interference between scattering contributions of neighboring particles, i.e., the structure factor. Here $\vec{q}$ is the scattering vector whose modulus is determined by the scattering angle $2 \Theta$ via $q$ $=4 \pi \sin \Theta / \lambda$, where $\lambda$ is the wavelength of the $x$-rays. For anisotropic particles, the scattering is further complicated by the fact that the interferences depend not only on the positional but also on the orientational correlations between the particles. Hence, $I(\vec{q})$ cannot be factorized into a product of the intra- and interparticle scattering (the form factor $F$ and structure factor $S$, respectively). For an isotropic phase of thin rods or platelets, however, the interparticle correlations are often weak. Therefore the approximation $S(q) \approx 1$ gives a sufficiently reasonable description of the SAXS intensity [30]. Treating the platelets as flat cylinders enables one to fully describe the platelets' orientation using their normal vector $\hat{e}_{\|}$. The scattered X-ray intensity can then be written as 


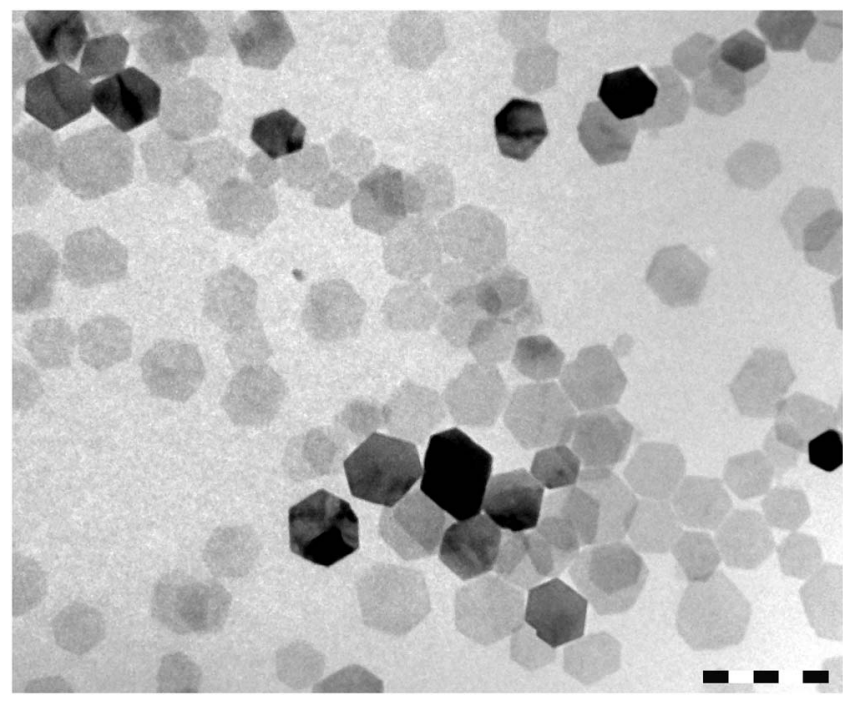

FIG. 2. Transmission electron micrograph showing the hexagonal gibbsite platelets used in this study. The average corner-tocorner diameter of the platelets is $232 \mathrm{~nm}$, with a polydispersity of about $20 \%$. The polymer layer is not visible on this micrograph; the scalebar denotes $500 \mathrm{~nm}$.

$$
I(\vec{q}) \propto\left\langle F\left(\vec{q}, \hat{e}_{\|}\right)\right\rangle,
$$

where the angular brackets denote ensemble averaging over all particles, with all different orientations.

Most of the SAXS data is collected at scattering vectors in the so-called "intermediate regime" [31], where $\frac{2 \pi}{D} \ll q \ll \frac{2 \pi}{L}$. In this domain, the scattering of a single platelet (the form factor $F$ ) is strongly directed along its normal. As shown in the Appendix, this leads to a simple and elegant approximate relation between the scattered intensity (as recorded in the SAXS patterns) and the ODF of the platelets

$$
I(q, \psi) \propto \frac{f(\psi)}{q^{2}},
$$

where $\psi$ describes the orientation of $\vec{q}$, see Fig. 1 . This relation implies that the ODF can be obtained directly from the scattering data at constant $q$ in the intermediate regime, making data analysis quite straightforward. However, as will be shown below, the approximations used in Eq. (7) can lead to a significant underestimate of the values of the order parameter $S_{2}$.

\section{EXPERIMENT}

\section{A. Sample preparation and characterization}

We synthesized hexagonal colloidal gibbsite $\left[\mathrm{Al}(\mathrm{OH})_{3}\right]$ platelets $[1,7]$ that were subsequently grafted with endfunctionalized polyisobutene and dispersed in toluene. Thus a model system of hard platelets is obtained, showing the liquid crystal phase transitions expected for hard-disk fluids $[3,5,32]$. Transmission electron microscopy (TEM), see Fig. 2 , and atomic force microscopy (AFM) were used to determine the average diameter $\langle D\rangle$ and thickness $\langle L\rangle$ of the dry particle core. The diameter of a platelet is measured as the average of the three corner-to-corner distances and the estimated thickness may contain a contribution of the collapsed steric stabilizer. We found $\langle D\rangle=232 \mathrm{~nm}$ and $\langle L\rangle=13 \mathrm{~nm}$ and a polydispersity of about $20 \%$ in both dimensions. In solution, the thickness of the sterically stabilizing polyisobutene brush is estimated to be 2 to $3 \mathrm{~nm}$, yielding effective dimensions of $D_{\text {eff }}=237 \mathrm{~nm}$ and $L_{\text {eff }}=18 \mathrm{~nm}$.

The dispersion of gibbsite platelets showed the first-order isotropic-nematic phase transition, with coexistence of the two phases at volume fractions $\phi_{I}=0.238$ and $\phi_{N}=0.255$. Volume fractions were calculated $[3,4]$ from the mass concentration, determined by drying a known amount of dispersion under nitrogen at $85^{\circ} \mathrm{C}$ to constant weight, and from the previously measured particle mass density $\rho_{p}=1.7 \mathrm{~g} / \mathrm{ml}$.

\section{B. Magnetic-field-induced birefringence}

In order to obtain an isotropic phase at isotropic-nematic coexistence, we brought the stock dispersion in the $I-N$ biphasic gap by dilution. After phase separation, which took 1 day, the amounts of isotropic and nematic phase were about $90 \%$ and $10 \%$, respectively. Both phases were extracted. The isotropic phase had a volume fraction of $\phi$ $=0.229$, which is slightly lower than $\phi_{I}=0.238$, probably due to fractionation effects (i.e., the nematic phase is enriched in more anisotropic disks, which is known to affect the volume fractions of both phases). It was used to prepare 11 samples in spectrophotometric cells with volume fractions ranging from $\phi=0.02$ to 0.246 , the latter value being just within the biphasic gap. In addition, a sample of pure solvent (toluene) was prepared in a $10.00-\mathrm{mm}$ cell for a background measurement. In spite of carefully sealing the suspension-filled cells with Teflon tape, evaporation of toluene could not be prevented, causing a shift of the samples' volume fraction to slightly higher values in time. We kept track of the actual volume fraction by weighing each sample before performing the birefringence measurements. The path length of the cells $d$ was chosen such that it increased from 1.00 to $10.00 \mathrm{~mm}$ with decreasing volume fraction to counterbalance the smaller birefringence at lower concentration.

The magnetic-field-induced birefringence was measured using a technique based on the modulation of the state of polarization of light [33]. This setup is suited to measure the linear birefringence $\Delta n$ very sensitively, as demonstrated by our measurement of the field-induced birefringence of toluene (the solvent).

The setup, similar to that described in Ref. [13], was equipped with (i) a green He-Ne laser $(\lambda=543.5 \mathrm{~nm})$, (ii) a vertical polarizer, (iii) a birefringent photoelastic modulator with its main optical axis at $45^{\circ}$ from the vertical direction and oscillating at a frequency $\nu=50 \mathrm{kHz}$ to avoid the influence of mechanical vibrations, (iv) the sample immersed in a horizontal magnetic field perpendicular to the light beam, (v) an analyzer at $45^{\circ}$ from the vertical direction, and (vi) a photomultiplier as a detector. A lock-in amplifier measured the component of the photomultiplier signal $I_{\nu}$ at the modulation frequency $\nu$, which is related to the birefringence $\Delta n$ by $I_{\nu}=I_{0} \sin [2 \pi \Delta n d / \lambda]$, where $I_{0}$ was kept constant and determined by calibration. The alternating magnetic field was 
generated by an electromagnet, sweeping with a triangular modulation from -0.8 to $0.8 \mathrm{~T}$. In order to ensure orientational equilibrium of the particles during measurements, the sweeping period was set to $49 \mathrm{~s}$, which is much larger than the typical response time of an isotropic solution to the magnetic field (measured to be in the range 10-100 ms, depending on volume fraction).

\section{Small-angle $x$-ray scattering}

From the stock dispersion of gibbsite platelets, four samples were prepared at volume fractions of $\phi=0.219$, $0,165,0.109$, and 0.050 . These samples were put in Lindemann glass capillaries (internal diameter $0.7 \mathrm{~mm}$ ) and subsequently flame sealed.

The SAXS setup of the Dutch-Belgian beamline BM-26 DUBBLE at the European Synchrotron Radiation Facility (ESRF, Grenoble, France) was used [34]. The scattered $\mathrm{x}$-rays were detected using a photon counting $13 \times 13 \mathrm{~cm}^{2}$ multiwire argon- $\mathrm{CO}_{2}$-gas-filled wire-chamber detector, with a pixel size of about $250 \mu \mathrm{m}$. An x-ray beam of $17 \mathrm{keV}$ (wavelength $\lambda=0.73 \AA$ ) and a sample-detector distance of about $8 \mathrm{~m}$ were used. The accessible $q$-range was $0.05-0.70 \mathrm{~nm}^{-1}$ both in the horizontal and vertical directions. The setup was calibrated using the fiber diffraction of wet rat-tail collagen, which has strong characteristic peaks at $q=2 \pi n / 67.2 \mathrm{~nm}^{-1}(n=1,3,5)$.

The beamline was equipped with a superconducting split coil magnet, giving a horizontally directed magnetic field and capable of reaching a maximum magnetic field of $B_{\max }$ $=7 \mathrm{~T}$ that was homogeneous to a level of $1 \%$ in a volume of $1 \mathrm{~cm}^{3}$. The magnetic field vector was perpendicular to the $\mathrm{x}$-ray beam. To avoid the influence of any sedimentation [35], the measurements were performed within 8 days after sample preparation. SAXS patterns were acquired at six different field strengths yielding equally spaced values of $B^{2}$ between 0 and $49 \mathrm{~T}^{2}$. Each pattern was corrected for detector sensitivity and background scattering.

\section{RESULTS AND DISCUSSION}

\section{A. Magnetic-field-induced birefringence}

First, we demonstrate the field-induced orientational order of the colloidal gibbsite platelets by showing the linear magnetic birefringence (of a different batch of suspension) as recorded with a digital camera (Nikon Coolpix 995) in Fig. 3. A biphasic sample, in a spectrophotometric cell with path length $d=2.00 \mathrm{~mm}$, was immersed in a horizontal magnetic field (perpendicular to the observation direction) and observed between crossed polarizers that made an angle of $45^{\circ}$ with the vertical. The isotropic phase on top, dark in zerofield, becomes bright under field. Moreover, from the increase of the birefringence of the isotropic phase with magnetic field, we can quickly obtain a very crude estimate of the field-induced birefringence. Using the approximate relation $\Delta=d \Delta n \approx \lambda_{\text {opt }} / 10$ [30] for the retardation $(\Delta)$ detection limit of the naked eye, with $\lambda_{\text {opt }} \approx 0.5 \mu \mathrm{m}$ the wavelength of the orange transmitted light, we obtain $|\Delta n| / B^{2} \approx 10^{-4} \mathrm{~T}^{-2}$.

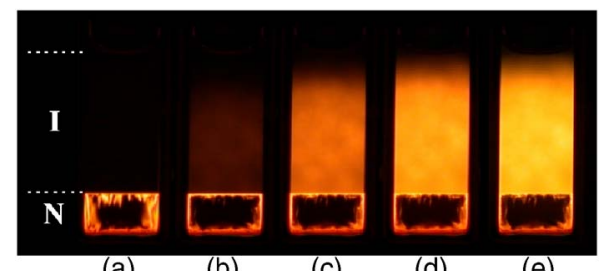

(a)

(b)

(c)

(e)

FIG. 3. (Color online) Illustration of the magnetic-field-induced birefringence in the isotropic top phase of the suspensions of sterically stabilized colloidal gibbsite platelets. An isotropic $(I)-$ nematic $(N)$ sample with path length $d=2.00 \mathrm{~mm}$ is placed between crossed polarizers in a horizontal magnetic field (a) 0 , (b) 0.5 , (c) 1 , (d) 1.5 , and (e) $2.0 \mathrm{~T}$, generated using a Bruker BE25v electromagnet. The sample is observed between crossed polarizers, making angles of $-45^{\circ}$ and $45^{\circ}$ with the vertical direction. As can be seen quite clearly, the birefringence in the isotropic phase increases with increasing magnetic field. The black patch in the nematic phase, which increases in size with the field strength, is caused by homeotropic anchoring of the platelets on the front and back glass walls of the cell. The birefringence gradient observed at the top of the sample is due to sedimentation of the particles.

With the experimental setup described in Sec. III B we have measured the field-induced birefringence, yielding $\Delta n$ vs $B$-curves, of which a typical one is shown in Fig. 4 . For weakly aligned suspensions, the field-induced-birefringence should be proportional to $B^{2}$ [17], which indeed appears to be the case: the $\Delta n$ vs $B$-curves are well described by second-order polynomials. The two samples of highest volume fractions showed some hysteresis due to the fact that they were slightly in the $I-N$ biphasic regime. However, this did not impede an accurate measurement of the field-induced birefringence at these concentrations.

The field-induced birefringence of toluene was measured as $\Delta n / B^{2}=2.5 \times 10^{-9} \mathrm{~T}^{-2}$. From this value the so-called Cotton-Mouton constant $K_{\mathrm{CM}}=\Delta n / \lambda B^{2}$ is obtained, where $\lambda$ is the wavelength at which it was determined, yielding $K_{\mathrm{CM}}=5 \times 10^{-3} \mathrm{~T}^{-2} \mathrm{~m}^{-1}$. From the literature values [36] of the Cotton-Mouton constants of benzene (7.84 $\left.\times 10^{-3} \mathrm{~T}^{-2} \mathrm{~m}^{-1}\right)$ and $p$-xylene $\left(8.17 \times 10^{-3} \mathrm{~T}^{-2} \mathrm{~m}^{-1}\right)$, mea-

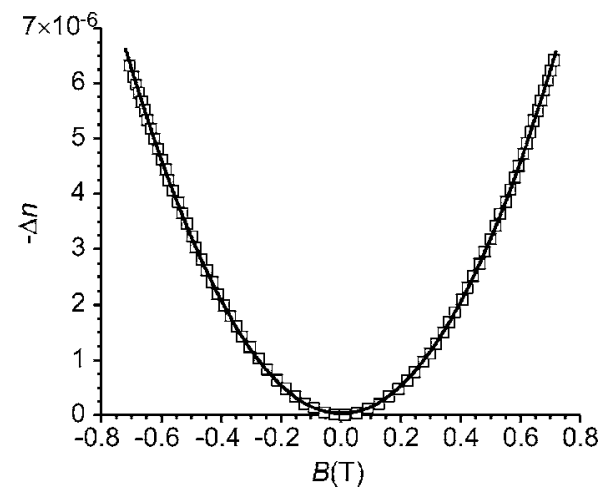

FIG. 4. Typical curve obtained from the measurement of the field-induced birefringence, in this case for a sample with $\phi$ $=0.219$ and $d=1.00 \mathrm{~mm}$. The open dots indicate measured data; the solid curve is a parabolic fit through the data, demonstrating that $-\Delta n \propto B^{2}$. 


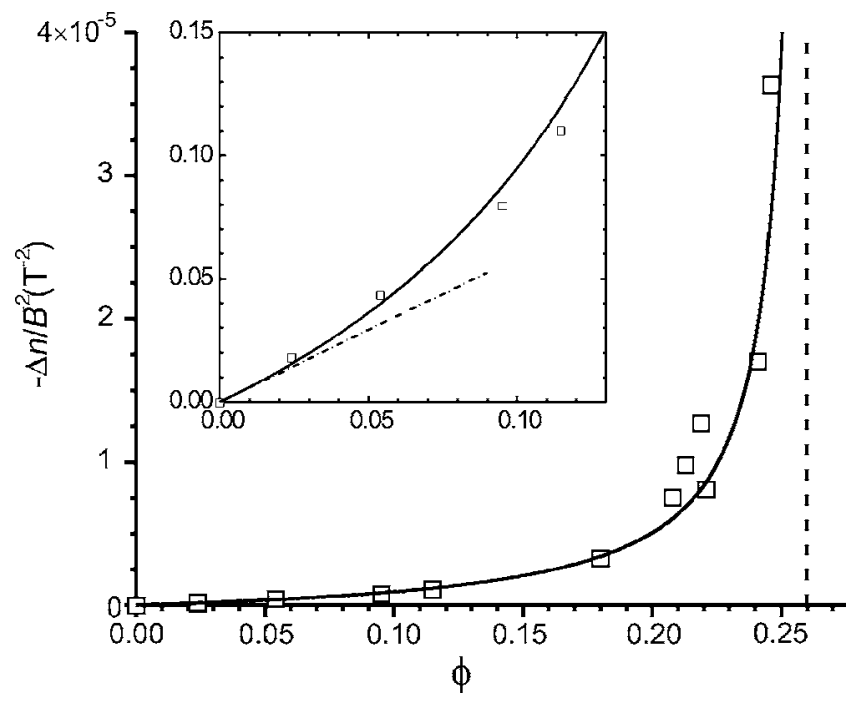

FIG. 5. The magnetic-field-induced birefringence $-\Delta n / B^{2}$ vs volume fraction (squares), and two-parameter fit (solid line). The field-induced birefringence diverges near the spinodal volume fraction $\phi^{*}$ indicated by the dashed line. The inset shows the low volume fraction region, with the dash-dotted line being the tangent to the fit at $\phi=0$, indicating ideal behavior (neglecting interparticle interactions). Apparently, the ODF [Eq. (9)] is able to describe the collective behavior almost up to $\phi^{*}$ very well.

sured at $\lambda=488 \mathrm{~nm}$, we estimate that of toluene (by interpolation and taking into account the different wavelength) at $7 \times 10^{-3} \mathrm{~T}^{-2} \mathrm{~m}^{-1}$ for our experimental wavelength and conclude that our measured value is slightly lower.

In Fig. 5 we show the magnetic-field-induced birefringence as a function of platelet volume fraction. For the coexisting isotropic phase, at $\phi=0.24$, the field-induced birefringence is about $2 \times 10^{-5} \mathrm{~T}^{-2}$. This is indeed of the same order of magnitude as the value we very crudely estimated above. The behavior observed in Fig. 5 is quite well described by Eq. (5), which allows us to extract the values of $\phi^{*}$ and $\Delta n_{\text {sat }} \Delta \chi$ through a two-parameter fit [13], yielding $\phi^{*}=0.26 \pm 0.03$ and $\Delta n_{\text {sat }} \Delta \chi=-3.6 \pm 0.1 \times 10^{-25} \mathrm{~J} / \mathrm{T}^{2}$.

In order to obtain $\Delta \chi$, we need to determine the specific birefringence $\Delta n_{\text {sat }}$ using Eq. (4). In other words, we can obtain $\Delta n_{\text {sat }}$ by measuring the birefringence $\Delta n$ of an isotropic or rather a monodomain nematic sample with known volume fraction and order parameter. We therefore prepared a biphasic suspension and rotated it in a magnetic field $(B$ $=1.4 \mathrm{~T})$ around its vertical axis, during which it phase separated. The nematic phase $\left(\phi_{N}=0.25\right)$, which initially consisted of numerous domains, coalesced completely to form a monodomain nematic phase with its director along the vertical axis (due to $\Delta \chi<0$ ), see Fig. 6. Using a retardation plate, we observed the so-called slow axis of the birefringent nematic phase to be oriented vertically. The bright green interference color that finally appears is a "second-order" green, with a retardation $\Delta=800 \pm 40 \mathrm{~nm}$ [37]. The sample thickness was $d=2.00 \mathrm{~mm}$; taking $S_{2}=0.7 \pm 0.2$ and using the relation $\Delta=d \Delta n$, and in Eq. (4) we find $\Delta n_{\text {sat }}=2.3 \pm 0.5$ $\times 10^{-3}$. This value agrees quite well with a measurement on a different sample using an optical birefringent compensator, yielding $\Delta n_{\text {sat }}=2.4 \pm 0.5 \times 10^{-3}$.

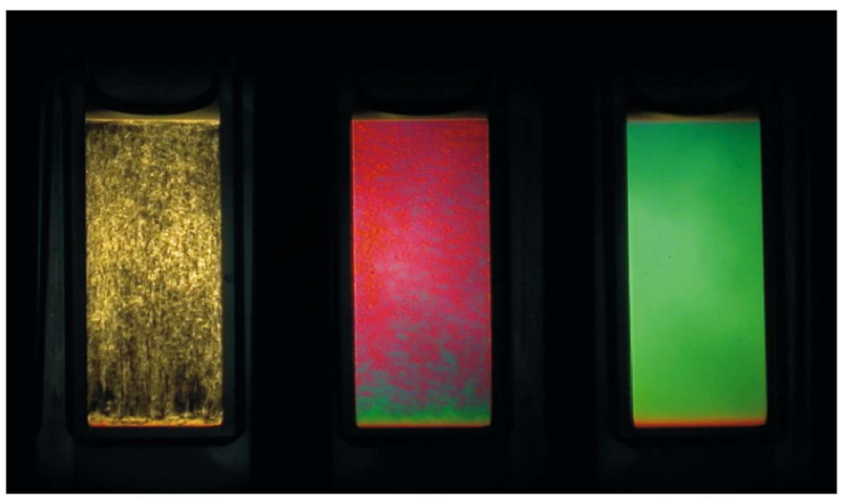

(a)

(b)

(c)

FIG. 6. (Color online) Almost completely nematic sample (i.e., with a very small amount of isotropic phase at the top) in a magnetic field, rotated around its vertical axis at an average rate of six revolutions per minute (a) just before starting rotation and after rotating for (b) 1 and (c) $2 \mathrm{~min}$. The magnetic field was directed horizontally, $B=1.4 \mathrm{~T}$, the polarizers made an angle of $-45^{\circ}$ and $45^{\circ}$ with the vertical direction. Due to this specific geometry, a (uniaxial) nematic phase is prepared with its director along the vertical rotation axis. Using an interference color chart [37], we find that the green interference color finally emerging is a "secondorder" green, with a retardation $\Delta=800 \pm 40 \mathrm{~nm}$.

We now obtain the diamagnetic susceptibility anisotropy of the gibbsite platelets $\Delta \chi=-1.6 \pm 0.6 \times 10^{-22} \mathrm{~J} / \mathrm{T}^{2}$, as measured with optical techniques. Using Eq. (3) we can now calculate the maximum field-induced orientational order in the isotropic phase, using $\phi=0.24, \phi^{*}=0.26, B=0.8 \mathrm{~T}$, and $\Delta \chi=-1.6 \times 10^{-22} \mathrm{~J} / \mathrm{T}^{2}$, as $S_{2}=-0.02$, which again confirms that we indeed deal only with weakly aligned states in this experiment.

\section{B. Small-angle $x$-ray scattering}

A striking feature of the gibbsite platelets, when placed in high magnetic fields, is the strong anisotropy of the scattering, shown in Fig. 7. The magnetic field is oriented perpendicular to the x-ray beam in the horizontal plane, see Fig. 1, hence this figure shows that the particles align with their normals perpendicular to the field (for platelike particles, the scattering extends to larger $q$ values in the direction parallel to the plate normal, see Fig. 14), confirming the negative value of $\Delta \chi$. In order to obtain this value from the data, we use the simple relation [Eq. (7)] between the scattered intensity and the ODF, derived in Sec. II C. The dependence with $q$ of the scattered intensity is shown in Fig. 8, where we show scattering profiles of an isotropic phase at zero field and at $B^{2}=40 \mathrm{~T}^{2}$. In the intermediate $q$-regime (which has an approximate range of $0.03 \mathrm{~nm}^{-1}<q<0.4 \mathrm{~nm}^{-1}$ ), this dependence is reasonably well described by a $q^{-2}$ decay. From Eq. (7) it follows that - in the intermediate regime-the ODF $f(\psi)$ is proportional to the azimuthal profile of the scattered intensity, at constant $q$. The ODF is obtained from the SAXS patterns by integrating over a small range of $q$-values $(\Delta q$ $\left.=0.003 \mathrm{~nm}^{-1}\right)$ to improve statistics and by making use of the centrosymmetry of the scattering according to 


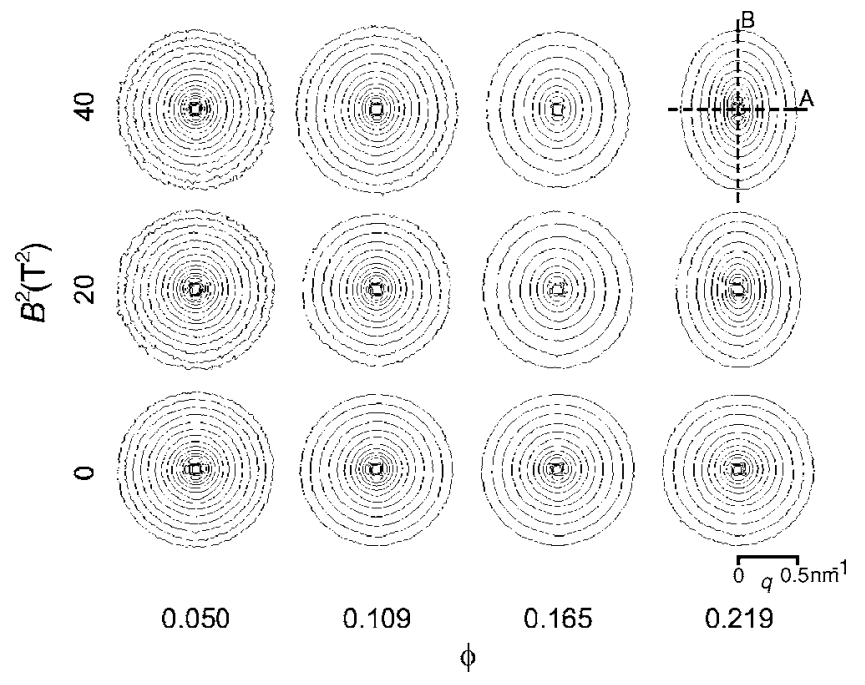

FIG. 7. Isointensity lines of a few of the small-angle $x$-ray scattering patterns recorded in this study, as a function of volume fraction $\phi$ and field strength squared $B^{2}$. The magnetic field is oriented horizontally. Clearly, the ellipticity of the scattering patterns increases with increasing volume fraction and field strength, while it decreases with increasing wave vector $q$, as more easily observed in Fig. 8. In the measurement of the ellipticity of the patterns, we have made use of two points $A$ and $B$ on the isointensity line; an example is shown in the upper right pattern.

$$
\begin{aligned}
I(\psi)= & \frac{1}{2} \int_{q_{i}-\Delta q}^{q_{i}+\Delta q} I(q, \psi) d q+\frac{1}{2} \int_{q_{i}-\Delta q}^{q_{i}+\Delta q} I(q, \psi+\pi) d q, \\
& 0<\psi<\pi .
\end{aligned}
$$

With appropriate normalization, these data simply represent the ODF, as shown in Fig. 9. The ODF given in Eq. (2) can be treated as if it were the result of a Taylor expansion, yielding

$$
f(\theta)=\frac{1}{Z} \exp \left[\frac{1}{3} \frac{\Delta \chi B^{2} P_{2}(\cos \theta)}{k T} \frac{1}{1-\phi / \phi^{*}}\right],
$$

which can be regarded as a "rescaled Boltzmann factor" and as the simplest ODF for interacting platelets at nonzero field

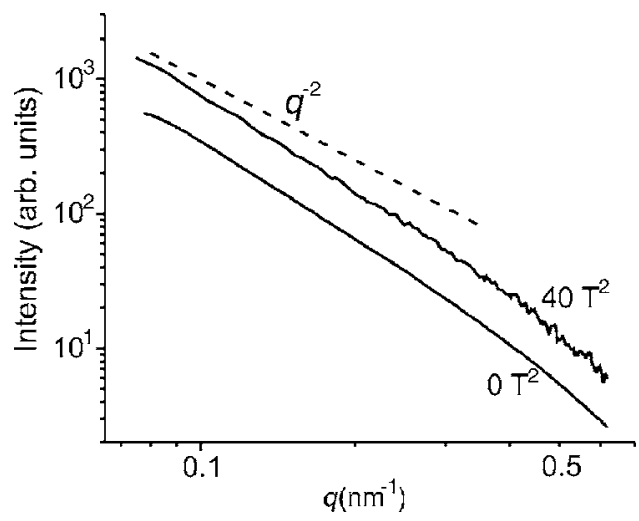

FIG. 8. Scattering profiles of the isotropic phase, $\phi=0.05$, at zero field, obtained by radial averaging, and at $B^{2}=40 \mathrm{~T}^{2}$, obtained for a thin slice (width $<0.01 \mathrm{rad}$ ) with $\psi=0$. The dashed line indicates that the scattered intensity approximately follows a $q^{-2}$ decay.

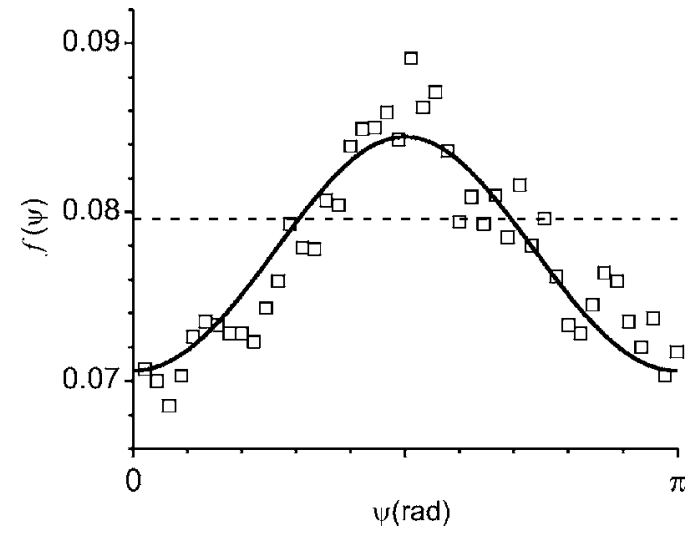

FIG. 9. An example of the $\operatorname{ODF} f(\psi)$ that was obtained from the SAXS patterns (of the same sample as used in Fig. $8, \phi=0.05, B^{2}$ $=40 \mathrm{~T}^{2}$, and $q=0.2 \mathrm{~nm}^{-1}$ ). The solid line is a fit using Eq. (11), yielding $K_{2}=-0.18$ and $S_{2}=-0.004$. The dashed line indicates the isotropic ODF $\left[f(\psi)=\frac{1}{4 \pi}\right]$.

strengths that correctly reverts to the limiting cases of "low field strength" $\left[B^{2} \ll 1\right.$, Eq. (2)] and of "noninteracting platelets" $\left(\phi / \phi^{*} \ll 1\right.$, yielding the ordinary Boltzmann factor).

Let us first only use the SAXS patterns at the lowest concentration $(\phi=0.05)$ to obtain $\Delta \chi$. The ODF (as presented in Fig. 9) is fitted using a two-parameter fit

$$
f(\psi)=K_{1} \exp \left[K_{2} \cos ^{2} \psi\right],
$$

where $K_{1}$ is a normalization constant, taken such that $\int f(\psi) d \Omega=1$ and

$$
K_{2}=\frac{1}{2} \frac{\Delta \chi B^{2}}{k T} \frac{1}{1-\phi / \phi^{*}} .
$$

We have performed this procedure for the scattering patterns of the sample at various magnetic fields, and at three intermediate $q$ values to obtain $K_{2}$ for $\phi=0.05$ as a function of $q$ and $B^{2}$. The resulting data are shown in Fig. 10. These data have been fitted for each $q$-value separately, as well as for

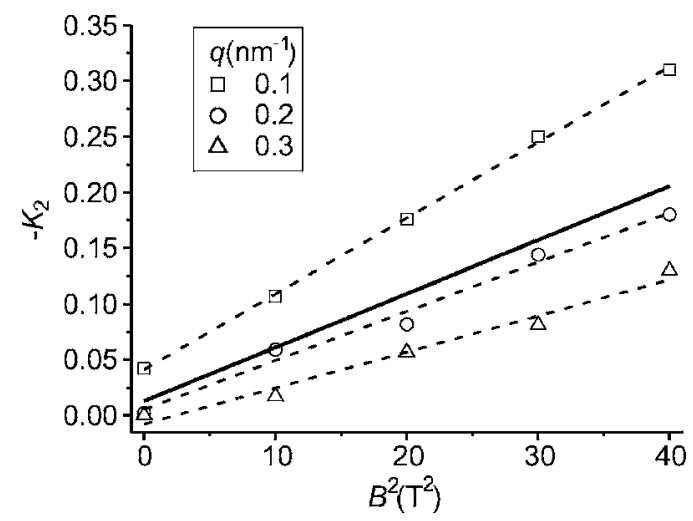

FIG. 10. Measurement of the ODF at low volume fraction $(\phi$ $=0.05)$ for three $q$-values in the intermediate regime. $K_{2}$ is related to the ODF through Eq. (10). The dashed lines indicate linear fits through the data at different $q$-values, while the solid line is a fit through all data points. $\Delta \chi$ is obtained from the slope of the solid line. 


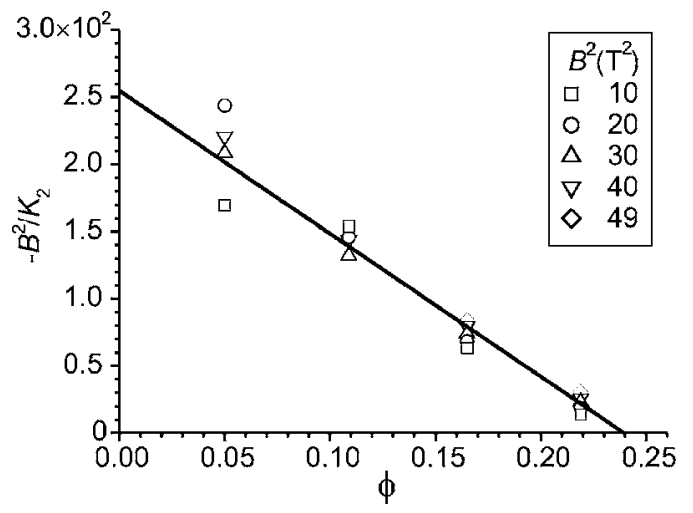

FIG. 11. Measurement of the ODF for the complete range of volume fractions and field strengths at $q=0.2 \mathrm{~nm}^{-1}$. The solid line is a linear fit, yielding $\Delta \chi=-3 \pm 1 \times 10^{-23} \mathrm{~J} / \mathrm{T}^{2}$ and $\phi=0.24 \pm 0.02$. The spread in the data, for different field strengths, is taken as an estimate of the error in this analysis.

the complete set using two-parameter linear fits, yielding the dashed lines and the solid one, respectively. From the figure it appears that the curves have a certain offset, which we attribute to a systematic experimental error. The effect appears stronger for lower $q$-values and for low volume fractions, and hence it is likely due to anisotropic parasitic scattering rather than from the sample itself, which leads us to ignore it and deliberately perform two-parameter fits. Furthermore, as can be seen in the figure, the data are better described in this way.

Taking $\phi^{*} \cong 0.25 \pm 0.02$ as an estimate for the spinodal concentration (it will be shown below that it is indeed a reasonable estimate) and using Eq. (11) we obtain $\Delta \chi=$ $-3 \pm 1 \times 10^{-23} \mathrm{~J} / \mathrm{T}^{2}$. The value of $\Delta \chi$ obtained here is proportional to the slope of the curves in Fig. 10 and therefore depends on the $q$-value where one measures the distribution function. However, the three $q$-values used $(0.1,0.2$, and $0.3 \mathrm{~nm}^{-1}$ ) cover the entire intermediate regime and we may therefore assume that the value of $\Delta \chi$ derived is reliable if we take the spread in the slopes (of the individual $q$-values) as an estimate of the error on $\Delta \chi$.

We now turn to an analysis of the complete set of data, i.e., we use the scattering patterns for all volume fractions $(0.05<\phi<0.219)$ and magnetic fields in order to obtain $\Delta \chi$ and $\phi^{*}$. Figure 10 shows that the slope of the solid line (a fit using all $q$-values) is close to that of the fit at $q=0.2 \mathrm{~nm}^{-1}$. Given the error in the slope, we proceed to perform the current complete analysis only for $q=0.2 \mathrm{~nm}^{-1}$ and treat the resulting values as representative for the intermediate $q$-regime. We have checked that this is the case for $\phi$ $=0.219$ as well.

In the same way as described above, the ODFs are obtained from the SAXS patterns and fitted using Eq. (10). Plotting $-B^{2} / K_{2}$ vs $\phi$ yields Fig. 11 . We take a linear fit through the data and obtain $\Delta \chi=-3 \pm 1 \times 10^{-23} \mathrm{~J} / \mathrm{T}^{2}$ and $\phi^{*}=0.24 \pm 0.02$, where the spread in the data gives an estimate of the error on the last two values. The value of $\Delta \chi$ obtained here agrees perfectly with that obtained at low volume fraction, validating the use of the "rescaled Boltzmann factor," Eq. (9). Furthermore, our earlier estimate of the spin-

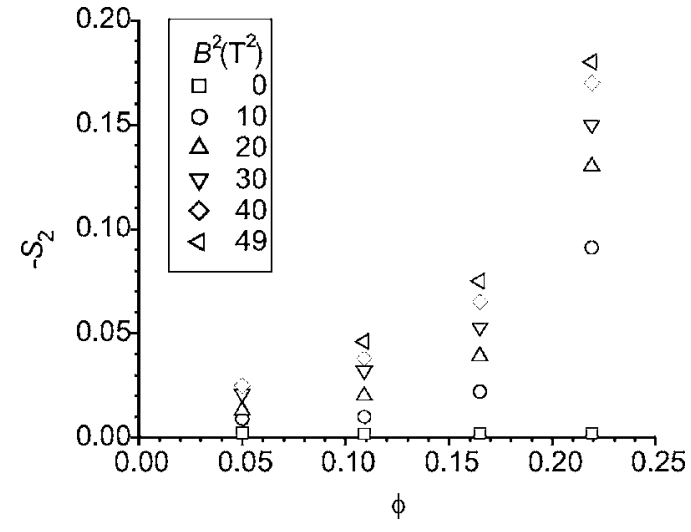

FIG. 12. The order parameter $S_{2}$ as calculated from the scattering patterns using Eq. (12).

odal volume fraction indeed agrees with the value obtained experimentally.

In order to evaluate the magnitude of the alignment, we calculate the order parameter from the scattering data, for each field strength and volume fraction. As we take discrete data points rather than an analytical form for the ODF, we use the following expression:

$$
\begin{aligned}
S_{2} & =2 \pi \int_{0}^{\pi} P_{2}(\cos \psi) f(\psi) \sin \psi d \psi \\
& \cong 2 \pi \frac{\pi}{n} \sum_{i=1}^{n} P_{2}\left(\cos \psi_{i}\right) f\left(\psi_{i}\right) \sin \psi_{i} .
\end{aligned}
$$

The calculated order parameters are shown in Fig. 12. For the most concentrated sample at highest field strength, $S_{2}$ $=-0.18$, which is about one-third of the maximum value $\left(-\frac{1}{2}\right)$ in this symmetry.

\section{GENERAL DISCUSSION AND CONCLUSION}

The present study describes two methods by which the magnetic-field-induced orientation of colloidal platelets in the isotropic phase was measured. Optical linear birefringence measurements were performed, and although it is a very sensitive technique to detect orientational ordering, it is only possible to obtain the order parameter $S_{2}$, i.e., the second moment of the ODF. Furthermore, it requires the knowledge of the saturation birefringence $\Delta n_{\text {sat }}$, for which a separate measurement is needed. The SAXS measurements are not as sensitive to phase anisotropy, however, using a simple model, they directly provide the orientational distribution function. Both techniques yield values of the diamagnetic susceptibility anisotropy $\Delta \chi$, although these values differ by a factor of about 5. A possible origin of this discrepancy could be the simplifications involved in the model describing the SAXS data. As shown in Sec. IV B, the model is able to qualitatively describe the magnetic-field-induced change of the SAXS pattern and allows for a straightforward data analysis. However, at a quantitative level, the approximations can lead to systematic errors. In particular, as is apparent from the Appendix, the model only accounts for the scat- 
tered intensity along the platelet normal. This very anisotropic axial intensity can contribute to the scattering pattern only if the normal of the particle is orthogonal to the x-ray beam, within the accuracy of order $\Delta \gamma$ (see the Appendix). For highly anisotropic particles with small aspect ratio $L / D, \Delta \gamma \ll 1$, therefore most particles do not obey this relation in a (nearly) isotropic suspension of platelets. This increases the role of the weak transverse scattering of the particles, which is neglected in the model. This effect should reduce the anisotropy of the SAXS pattern, which is thus overestimated in our simple model. As a result, the model underestimates the value of $\Delta \chi$. One can show that for $L / D=1 / 18$, approximately $20 \%$ of the total scattering intensity is not taken into account in the model, in a rather broad range of $q$ values obeying the relation $\frac{2 \pi}{D} \ll q \ll \frac{2 \pi}{L}$. Even more severe discrepancies arise if $q$ approaches one of the limits in the inequality relation, $\frac{2 \pi}{D}=0.027 \mathrm{~nm}^{-1}$ and $\frac{2 \pi}{L}$ $=0.48 \mathrm{~nm}^{-1}$, respectively. Due to the restriction on the smallest $q$-value in our data, the analysis is performed for $q$ in the range $0.1-0.3 \mathrm{~nm}^{-1}$. While these values obey the $\frac{2 \pi}{D} \ll q$ inequality reasonably well, the second inequality $q \ll \frac{2 \pi}{L}$ is less secure, especially at the largest analyzed $q$ values. As a result, the unaccounted component of the SAXS intensity, which reduces the scattering anisotropy, plays a more important role at larger $q$. This explains the $q$-dependence illustrated in Fig. 10. We have performed a few model calculations with an explicit expression for the scattering of discotic particles [Eq. (A2)]. These calculations indicate that the simple model underestimates $\Delta \chi$ by about a factor of 2 to 3 depending on the $q$ value. In view of the above, we are led to conclude that the diamagnetic susceptibility anisotropy of our colloidal gibbsite platelets has an order of magnitude $\Delta \chi \sim 10^{-22} \mathrm{~J} / \mathrm{T}^{2}$. This estimate is much lower than the value reported for goethite nanorods because these latter particles bear spins due to the Fe(III) ions. Nevertheless, the $\Delta \chi$ of gibbsite platelets is at least an order of magnitude larger than those of suspensions of organic nanoparticles, even though the $\mathrm{Al}(\mathrm{III})$ ions do not bear any spins. This point illustrates the interest of mineral colloidal suspensions of anisotropic particles over their organic counterparts, as far as electronic properties are concerned [38]. The influence of a magnetic field on the nematic phase of the suspensions of gibbsite platelets is presently under study in our laboratories.

\section{ACKNOWLEDGMENTS}

The authors thank Bonny Kuipers for support with the Bruker magnet, Geert Rikken for providing the superconducting magnet, Peter van der Linden for the room temperature insert of the magnet, and I. P. Dolbnya for aligning the beamline optics for the SAXS experiment. The Netherlands Organisation for Scientific Research (NWO) is thanked for financing the project of D.B. and for granting beamtime at DUBBLE. D.B. furthermore thanks the European Marie $\mathrm{Cu}-$ rie Training Site Program for Contract No. HPMT-CT-200000047 that made his stay at the Université Paris-Sud possible.

\section{APPENDIX}

Using the $x y z$ Cartesian coordinates introduced in Fig. 1, the platelet normal $\hat{e}_{\|}$takes the form

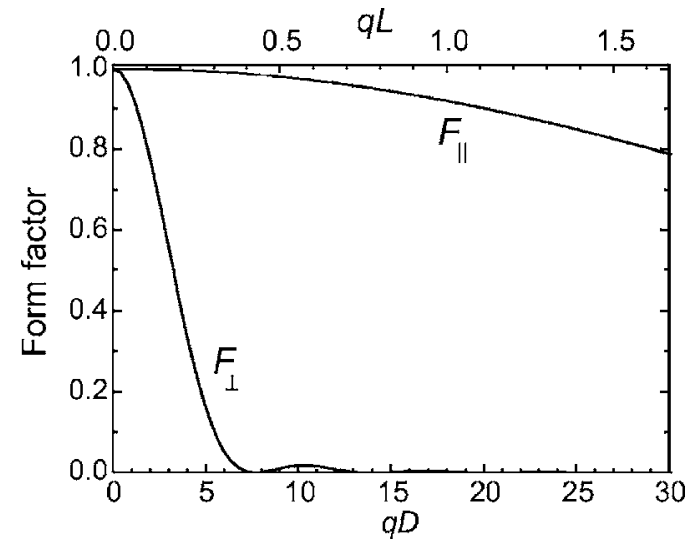

FIG. 13. The two components of the form factor of a cylindrical disk with aspect ratio $L / D=1 / 18$, as defined in Eq. (A2). In the intermediate regime, $F_{\perp}$ decays very fast, while $F_{\|}$decreases only slowly with $q$.

$\hat{e}_{\|}=(\sin \theta \cos \varphi, \sin \theta \sin \varphi, \cos \theta)$. In the following, we evaluate the scattered intensity, Eq. (6), at a given position on the detector, i.e., for a given value of the scattering vector $\vec{q}=\left(q_{x}, 0, q_{z}\right)=q\left(\sin \psi_{0}, 0, \cos \psi_{0}\right)$, where small-angle approximation $\left(q_{y} \cong 0\right)$ is used. For an ensemble of platelets with orientational distribution function $f(\theta)$, Eq. (6) can be written as

$$
I(q, \psi) \propto\left\langle F\left(\vec{q}, \hat{e}_{\|}\right)\right\rangle=\iint f(\theta) F(\vec{q}) \sin \theta d \theta d \varphi .
$$

The form factor of a cylinder can be written as the product of two components [31]

$F\left(q_{\|}, q_{\perp}\right)=F_{\|}\left(q_{\|}\right) F_{\perp}\left(q_{\perp}\right)=\left[\frac{\sin \left(\frac{1}{2} q_{\|} L\right)}{\frac{1}{2} q_{\|} L}\right]^{2}\left[\frac{J_{1}\left(\frac{1}{2} q_{\perp} D\right)}{\frac{1}{2} q_{\perp} D}\right]^{2}$,

where $J_{1}$ is the Bessel function of the first kind and

$$
\begin{aligned}
& q_{\|}=q \cos \gamma, \\
& q_{\perp}=q \sin \gamma
\end{aligned}
$$

are two orthogonal components of the scattering vector parallel and perpendicular to the platelet normal $\hat{e}_{\|}$, respectively, which are described by the angle $\gamma$ between the particle normal $\hat{e}_{\|}$and the wave vector $\vec{q}$. Figure 1 illustrates the experimental configuration and the used symbols. $\gamma$ is related to $\theta$ and $\phi$ (defining the platelet orientation with respect to the $x$-axis), and $\psi$ (that defines the orientation of the scattering vector in the $x z$-plane) according to

$$
\cos \gamma=\cos \theta \cos \psi+\sin \theta \sin \psi \cos \phi .
$$

Figure 13 depicts the two components $F_{\|}(q)$ and $F_{\perp}(q)$ of the form factor of a cylindrical disk with aspect ratio $L / D$ $=1 / 18$. (Here, we consider the aspect ratio of the gibbsite core of the platelets because the electron density contrast between the steric stabilizer layer and the solvent is negli- 

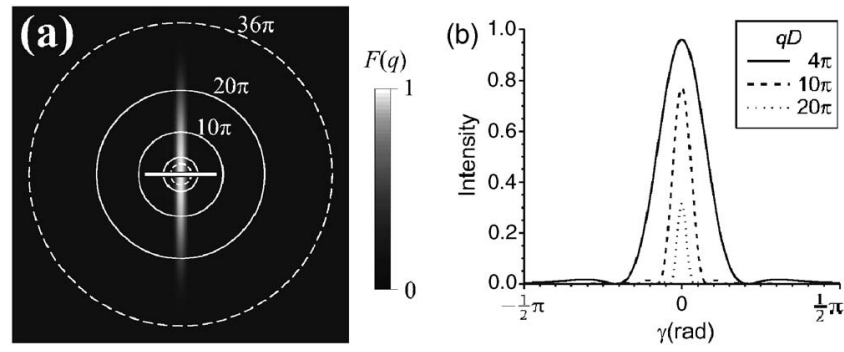

FIG. 14. The scattering of a platelike particle is strongly directed along its normal, as demonstrated by (a) the form factor $F(q)$ of a platelike particle with $L / D=1 / 18$. As this is a planar cut through the three-dimensional Fourier space, the central peak is in fact a three-dimensional ellipsoidlike object rather than a twodimensional ellipselike object. For clarity, a disk with the same aspect ratio and analogous orientation is depicted in the scattering pattern. For three circles, the corresponding $q D$-value is given. The dashed circles give the extremes of the intermediate regime; the solid circles indicate the azimuthal paths along which we calculate the scattered intensity that is shown in (b) for $q D=4 \pi, 10 \pi$, and $20 \pi$.

gible.) As can be seen in Fig. 13, for $q$ values obeying the relation $\frac{2 \pi}{D} \ll q \ll \frac{2 \pi}{L}, F_{\|}(q)$ and $F_{\perp}(q)$ differ substantially in magnitude. Most of the scattering is contained in this "intermediate regime" [31]. Here $F_{\|}(q)$ remains nearly constant, while $F_{\perp}(q)$ decays to values one to three orders of magnitude lower than $F_{\|}(q)$. Thus, for a given value of $q$, scattering essentially comes only from platelets oriented so that $q_{\perp}$ is small, i.e., for small angles of $\gamma$. Hence, we have a very narrow, ellipsoidlike axial scattering peak where most of the scattering is found, as illustrated in Fig. 14.

For weakly perturbed orientational distributions, $f(\theta)$ varies slowly over the narrow scattering peak. This allows us to take it out of the integral to obtain

$$
I(q, \psi) \propto f(\theta=\psi) \iint F_{\|}\left(q_{\|}\right) F_{\perp}\left(q_{\perp}\right) \sin \theta d \theta d \varphi .
$$

We further approximate $F_{\|}\left(q_{\|}\right) \approx 1$ and

$$
F_{\perp}\left(q_{\perp}\right) \approx \begin{cases}1, & q_{\perp}<q_{0} \\ 0, & q_{\perp}>q_{0},\end{cases}
$$

where $q_{0}=3.83 / \frac{1}{2} D$ is the position of the first minimum of $F_{\perp}$. This is equivalent to the requirement that $\gamma<\Delta \gamma$, where $\Delta \gamma(q)=\arctan \left(q_{0} / q\right) \approx q_{0} / q$. An exact evaluation of Eq. (A5) shows that about $80 \%$ of the scattering originates from the particles with $\gamma<\Delta \gamma$. The remaining 20\% (which are neglected within the current model) comes from particles with $\gamma>\Delta \gamma$. For the particles under consideration (with $L / D$ $=1 / 18), \Delta \gamma$ is approximately $8^{\circ}$ at $q=\pi / L$, demonstrating that we indeed have a very narrow scattering peak. These approximations allow us to evaluate the integral in Eq. (A5) to

$$
\iint F_{\|}\left(q_{\|}\right) F_{\perp}\left(q_{\perp}\right) \sin \theta d \theta d \varphi \approx(\Delta \gamma)^{2} \propto \frac{1}{q^{2}} .
$$

By substituting this equation into Eq. (A5), the result in the main text [Eq. (7)] is obtained.
[1] A. M. Wierenga, T. A. J. Lenstra, and A. P. Philipse, Colloids Surf., A 134, 359 (1998).

[2] J. E. G. J. Wijnhoven, Chem. Mater. 16, 3821 (2004); C. Vonk, S. M. Oversteegen, and J. E. G. J. Wijnhoven, J. Colloid Interface Sci. 287, 521 (2005); J. E. G. J. Wijnhoven, ibid. 292, 403 (2005).

[3] F. M. van der Kooij and H. N. W. Lekkerkerker, J. Phys. Chem. B 102, 7829 (1998).

[4] F. M. van der Kooij, D. van der Beek, and H. N. W. Lekkerkerker, J. Phys. Chem. B 105, 1696 (2001).

[5] F. M. van der Kooij, K. Kassapidou, and H. N. W. Lekkerkerker, Nature (London) 406, 868 (2000); D. van der Beek, A. V. Petukhov, S. M. Oversteegen, G. J. Vroege, and H. N. W. Lekkerkerker, Eur. Phys. J. E 16, 253 (2005).

[6] D. van der Beek and H. N. W. Lekkerkerker, Europhys. Lett. 61, 702 (2003); A. V. Petukhov, D. van der Beek, R. P. A. Dullens, I. P. Dolbnya, G. J. Vroege, and H. N. W. Lekkerkerker, Phys. Rev. Lett. 95, 077801 (2005).

[7] D. van der Beek and H. N. W. Lekkerkerker, Langmuir 20, 8582 (2004).

[8] J. Kerr, Report of the Annual Meeting of the British Association for the Advancement of Science, p. 568 (1901).

[9] Q. Majorana, C. R. Hebd. Seances Acad. Sci. 135, 159 (1902); 135, 235 (1902); Rend. Accad. Naz. Lincei 11-1, 374 (1902); 11-1, 463 (1902); 11-1, 531 (1902); 11-2, 90 (1902); 11-2,
139 (1902)

[10] A. Cotton and H. Mouton, C. R. Hebd. Seances Acad. Sci. 141, 317 (1905); 141, 349 (1905); 142, 203 (1906); Ann. Chim. Phys. 11, 145 (1907); 11, 289 (1907).

[11] A. Cotton and H. Mouton, C. R. Hebd. Seances Acad. Sci. 145, 229 (1907).

[12] B. J. Lemaire, P. Davidson, J. Ferré, J. P. Jamet, P. Panine, I. Dozov, and J. P. Jolivet, Phys. Rev. Lett. 88, 125507 (2002).

[13] B. J. Lemaire, P. Davidson, J. Ferré, J. P. Jamet, D. Petermann, P. Panine, I. Dozov, and J. P. Jolivet, Eur. Phys. J. E 13, 291 (2004).

[14] B. J. Lemaire, P. Davidson, D. Petermann, P. Panine, I. Dozov, D. Stoenescu, and J. P. Jolivet, Eur. Phys. J. E 13, 309 (2004).

[15] J. Sugiyama, H. Chanzy, and G. Maret, Macromolecules 25, 4232 (1992).

[16] S. Fraden, G. Maret, D. L. D. Caspar, and R. B. Meyer, Phys. Rev. Lett. 63, 2068 (1989).

[17] S. Fraden, G. Maret, and D. L. D. Caspar, Phys. Rev. E 48, 2816 (1993).

[18] H. Nakamura and K. Okano, Phys. Rev. Lett. 50, 186 (1983); 50, 1534 (1983).

[19] J. Tang and S. Fraden, Phys. Rev. Lett. 71, 3509 (1993); C. Martin, H. Kramer, C. Johner, B. Weyerich, J. Biegel, R. Deike, M. Hagenbüchle, and R. Weber, Macromolecules 28, 3175 (1995). 
[20] J. Torbet and G. Maret, Biopolymers 20, 2657 (1981).

[21] N. E. Geacintov, F. van Nostrand, M. Pope, and J. B. Tinkel, Biochim. Biophys. Acta 226, 486 (1971).

[22] K. Muta, H. Takezoe, A. Fukuda, and E. Kuze, Jpn. J. Appl. Phys. 18, 2073 (1979).

[23] T. W. Stinson and J. D. Litster, Phys. Rev. Lett. 25, 503 (1970).

[24] G. Maret, M. v. Schickfus, A. Mayer, and K. Dransfeld, Phys. Rev. Lett. 35, 397 (1975).

[25] P. Photinos, C. Rosenblatt, T. M. Schuster, and A. Saupe, J. Chem. Phys. 87, 6740 (1987).

[26] J. P. Straley, Mol. Cryst. Liq. Cryst. 22, 333 (1973).

[27] J. Zadoc-Kahn, C. R. Hebd. Seances Acad. Sci. 190, 672 (1930); J. Zadoc-Kahn Eisenmann, Ann. Phys. (Paris) 6, 455 (1936); P. G. de Gennes, Phys. Lett. 30A, 454 (1969); G. K. L. Wong and Y. R. Shen, Phys. Rev. Lett. 30, 895 (1972); P. G. de Gennes, The Physics of Liquid Crystals (Clarendon Press, Oxford, 1974).

[28] S. Nevue-Prin, F. A. Tourinho, J.-C. Bacri, and R. Perzynski, Colloids Surf., A 80, 1 (1993).

[29] J. B. Hayter and J. Penfold, J. Phys. Chem. 88, 4589 (1984); S. M. Clarke, A. R. Rennie, and P. Convert, Europhys. Lett. 35, 233 (1996); I. W. Hamley, G. R. Luckhurst, R. M.
Richardson, and F. Santos, J. Chem. Phys. 116, 3887 (2002); M. H. Li, A. Brûlet, P. Davidson, P. Keller, and J. P. Cotton, Phys. Rev. Lett. 70, 2297 (1993); P. Davidson, D. Petermann, and A. M. Levelut, J. Phys. II 5, 113 (1995).

[30] B. J. Lemaire, P. Panine, J. C. P. Gabriel, and P. Davidson, Europhys. Lett. 59, 55 (2002).

[31] A. Guinier and G. Fournet, Small Angle Scattering of X-rays (Wiley, New York, 1955).

[32] J. A. C. Veerman and D. Frenkel, Phys. Rev. A 45, 5632 (1992).

[33] J. Ferré and G. A. Gehring, Rep. Prog. Phys. 47, 513 (1984)

[34] W. Bras, I. P. Dolbnya, D. Detollenaere, R. van Tol, M. Malfois, G. N. Greaves, A. J. Ryan, and E. Heeley, J. Appl. Crystallogr. 36, 791 (2003).

[35] D. van der Beek, T. Schilling, and H. N. W. Lekkerkerker, J. Chem. Phys. 121, 5423 (2004).

[36] M. Surma and M. Ciszek, Physica B 228, 295 (1996).

[37] F. D. Bloss, An Introduction to the Methods of Optical Crystallography (Holt, Rinehart and Winston, New York, 1961).

[38] J. C. P. Gabriel and P. Davidson, Adv. Mater. (Weinheim, Ger.) 12, 9 (2000); Top. Curr. Chem. 226, 119 (2003); P. Davidson and J. C. P. Gabriel, Curr. Opin. Colloid Interface Sci. 9, 377 (2005). 